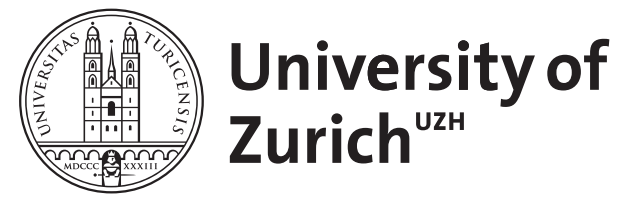

Zurich Open Repository and Archive

University of Zurich

University Library

Strickhofstrasse 39

CH-8057 Zurich

www.zora.uzh.ch

Year: 2006

Can the prospective and retrospective memory questionnaire (PRMQ) predict actual prospective memory performance?

Kliegel, Matthias ; Jäger, Theodor

DOI: https://doi.org/10.1007/s12144-006-1002-8

Posted at the Zurich Open Repository and Archive, University of Zurich

ZORA URL: https://doi.org/10.5167/uzh-156418

Journal Article

Published Version

Originally published at:

Kliegel, Matthias; Jäger, Theodor (2006). Can the prospective and retrospective memory questionnaire (PRMQ) predict actual prospective memory performance? Current Psychology, 25(3):182-191.

DOI: https://doi.org/10.1007/s12144-006-1002-8 


\title{
Can the Prospective and Retrospective Memory Questionnaire (PRMQ) Predict Actual Prospective Memory Performance?
}

\author{
MATTHIAS KLIEGEL \\ University of Zurich
}

THEODOR JÄGER

Saarland University

$\mathrm{T}$

he present study investigated the relationship between individual scores in the recently developed Prospective and Retrospective Memory Questionnaire (PRMQ) and measures of actual prospective memory test performance. Standard laboratory time-based and event-based prospective memory tasks were administered to a sample of 87 adults from Switzerland (age: $M=44.11, S D=18.94 ; 47$ females). We investigated how actual prospective memory performance is related to the scores of the PRMQ. Furthermore, we examined the relationship between prospective memory performances and self-reports of general metamemory judgments (that is, beliefs about and the use of one's memory abilities). The most important finding was that the different subscales of the PRMQ seem to have a differential sensitivity in the prediction of actual task performance: Prospective memory performance was predicted by the prospective memory subscales of the PRMQ, but not by the retrospective memory subscales. Furthermore, distinct aspects of metamemory were found to relate to actual prospective memory performance and to the scores of the PRMQ, providing crossvalidation for the PRMQ. In sum, the present study extends initial reports supporting the validity of the PRMQ and provides first evidence for the utility of the PRMQ subscales in differentiating between prospective and retrospective memory task performance.

A highly relevant everyday memory process that has only recently been given increased attention is the ability to remember to perform previously planned activities, referred to as prospective memory (PM; see Brandimonte, Einstein, \& McDaniel, 1996; Ellis \& Kvavilashvili, 2000). PM tasks - such as remembering to pack the passport before going on the airport-concern the self-initiated retrieval of intentions at a specific moment and are contrasted with retrospective memory (RM) tasks. RM tasks involve the externally prompted retrieval of information content such as remembering previously studied foreign language words in a vocabulary test (for example, Baddeley, 1990). 
The literature has subdivided PM tasks into event-based tasks, in which the execution of an intended action is triggered by a particular event, and into time-based tasks, which require remembering to perform the intended action at a specific point in time, or after a specified period of time has elapsed (Einstein et al., 1995; Kliegel et al., 2001). The appropriate instance for carrying out the intended action is always embedded within ongoing activities, referred to as the ongoing task.

While the vast majority of research on PM has investigated mean level differences in PM test performance across groups or experimental manipulations, few studies have sought to gain insight into PM by using questionnaires aimed to provide estimates of an individual's ability to carry out intended activities. The most prominent example of such self-rating measures is the recently developed Prospective and Retrospective Memory Questionnaire (PRMQ; Crawford et al., 2006; Smith et al., 2000). The PRMQ was designed to disentangle self-rated PM and RM performance in everyday life or in clinical settings. Previous self-reports of memory ability ignored the distinction between PM and RM tasks. Specifically, the PRMQ assesses how often errors in everyday PM or RM tasks occur. Factor analyses revealed that a tripartite structure model best fits the PRMQ scores, that is, a general memory factor and two orthogonal specific factors of PM and RM (Crawford et al., 2003). These factors are captured using sixteen items that are equally divided between a PM subscale assessing everyday PM errors, and a RM subscale assessing everyday RM errors.

The proposed narrow relationship between the PRMQ scores and objective measures of PM performance remains to be empirically tested, however, in order to examine the validity of the PRMQ as an instrument reliably predicting an individual's actual memory performance. To the best of our knowledge, only one published study has started to relate PRMQ scores to performance-based memory measures: Mäntylä (2003) recruited participants reporting a high degree of everyday memory problems (that is, self-reporters) and participants reporting no particular memory problems (that is, nonreporters). Mäntylä found that self-reporters had generally higher scores in the PRMQ, indicating more frequent everyday memory problems among the self-reporters relative to the nonreporters. The self-reporters also exhibited diminished performance in PM tasks relative to the nonreporters. In Experiment 2 of Mäntylä's study, the PM Scale of the PRMQ was significantly correlated with performance in a single-trial laboratory-based PM task, providing initial validation data for the PRMQ. The RM Scale of the PRMQ, however, was also significantly correlated with performance in the PM task to a similarly high degree as the PM Scale. In conclusion, in the study by Mäntylä, the PM Scale and the RM Scale of the PRMQ do not seem to provide differential sensitivity for predicting actual PM performance, which is unexpected with regard to the a priori difference between a PM subscale and a RM subscale within the PRMQ instrument.

Based on this outcome, the authors of the present study set out to further examine the validity of the PRMQ by exploring how the scores of the PRMQ relate to performance measures of two standard multiple-response laboratory-based PM tests. While in the study of Mäntylä (2003; Experiment 2) the relation between the PRMQ and actual PM performance was examined for only one (event-based) PM task, in the 
present study participants completed two parallel PM tasks in the laboratory comprising an event-based and a time-based PM task. Moreover, we administered PM tasks that both included five PM trials, and thus should have a higher reliability than the single-trial PM task applied in the study of Mäntylä (see Maylor, 1993, 1996, for discussions). Our examination aimed to reveal whether the PRMQ is valid in the sense that it translates into actual PM task performance. Importantly, the usefulness of the PRMQ in distinguishing between PM and RM performance by its separate scales would be supported if the PM Scale predicted PM task performance better than the RM Scale.

We also aimed to examine the relationship between PM performance and individual differences in a standard metamemory questionnaire. As metamemory involves memory strategy use, knowledge about how memory functions, or memory self-efficacy, that is, beliefs about one's own memory ability (Dixon, 1989; Hultsch et al., 1988), general metamemory beliefs can be assumed to be related to actual PM performance as well. In a previous study, McDonald-Miszczak, Gould, and Tychynski (1999) used singletrial time-based and event-based PM tasks and found that memory self-efficacy predicted both time-based and event-based PM performance. This finding is especially appealing since memory self-efficacy is supposed to influence behavior in a memorytask situation. The present study aimed to further elucidate the relationship between distinct aspects of metamemory as assessed by the Metamemory in Adulthood Questionnaire (MIA; Dixon \& Hultsch, 1984; Dixon, Hultsch, \& Hertzog, 1988) and actual performance in laboratory-based PM tasks.

Finally, since in the present study, for the first time, both the complete PRMQ and the MIA were administered within the same sample, we sought to cross-validate the PRMQ with the well-established MIA. The motivation for this examination comes from the idea that self-reported memory errors may well relate to specific aspects of metamemory. For instance, a greater extent of self-reported everyday PM or RM errors may be associated with feelings of low capacity of one's memory, with heightened anxiety in memory situations, or with increased use of memory strategies in order to prevent such memory errors.

To sum up, in the present study we aimed at investigating the predictive value of the PRMQ and the MIA for actual PM task performance and we cross-validated the PRMQ with the established MIA instrument. By this means, we sought to further document the validity of the PRMQ for its suggested usefulness in providing estimates of actual PM and RM performance among healthy normal adults or clinical patients reporting diminished cognitive abilities.

\section{METHOD}

\section{Participants}

A sample of 87 volunteers participated in this study (age: $M=44.11, S D=18.94$; 47 females), who were recruited through newspaper ads as well as randomly selected from the department participant pool. All participants were Caucasian and community- 
dwelling adults who lived within the region of Zurich, Switzerland. The equivalent of eight U.S. dollars was paid to every participant. Institutional human subject review permission was granted to protect the welfare of participants.

\section{Materials}

\section{PM Tasks}

(a) Ongoing Task. We used the n-back working memory task as the ongoing task in which both the event-based and the time-based PM task were embedded (two different versions of the n-back task were administered for every PM task). In n-back tasks, participants are typically presented with a series of stimuli and are to indicate whether the current stimulus matches the stimulus presented $\mathrm{n}$ stimuli back in the series, whereby $n$ typically equals a number between one and three. In the present study, we used a two-back version of this task: Participants viewed pseudo-random sequences of the Snodgrass and Vanderwart (1980) pictures on a computer screen, each displayed for four seconds (s) with one $s$ inter-stimulus interval. Instructions were given to press a "yes" key if the picture was the same as that which occurred twice before, otherwise a "no" key had to be pressed. The n-back task consisted of one-hundred twenty-two trials (maximum hits $=40$ ). The number of correct responses was obtained by adding correct rejections and hits for each participant.

\section{(b) PM Component.}

(1) Event-based. The event-based PM task was to remember to press a target key whenever a picture depicting an animal appeared during the n-back task. There were five PM targets, which occurred at 1:50, 3:50, 5:50, 7:50, and 9:50 minutes after the start of the ongoing task in order to closely parallel the occurrence of the event-based PM targets to the time-based PM target times (see below). Every hit on the target key that occurred within five s after the presentation of a PM target was scored as PM hit (range equals zero to five).

(2) Time-based. The time-based PM task was to remember to press a target key at two-minute intervals from the start of the n-back task as accurately as possible. To monitor the time, participants could press the "space" key to see a time counter clock ["00:00"] which appeared for three s. Every hit on the target key that occurred within a time window of five s ( $\pm 2.5 \mathrm{~s}$ ) around the PM target times (Kliegel et al., 2001, 2005) was scored as PM hit (range equals zero to five).

$P R M Q$. The Prospective and Retrospective Memory Questionnaire (PRMQ; Crawford et al., 2003, 2006; Smith et al., 2000) was used to assess the frequency of self-reported everyday PM and RM errors. The PRMQ consists of sixteen items equally divided between a PM and a RM subscale. On a 5-point Likert scale, participants rated how often each type of memory failure occurs in their everyday life $(5=$ very often; $1=$ 
never). Higher scores in the PRMQ subscales indicate a higher incidence of memory errors or memory problems.

MIA. We used a shortened 74-item version (Ponds \& Jolles, 1999) of the Metamemory in Adulthood Questionnaire (MIA; Dixon \& Hultsch, 1984; Dixon et al., 1988), which consists of seven scales (each scale's name and number of items is given in parenthesis below).

The seven scales provide information about an individual's knowledge of basic memory processes and tasks (Task; 10), self-rated memory capacity (Capacity; 12), perceptions of memory stability versus decline over the last ten years (Change; 10), anxiety regarding memory situations (that is, the degree of discomfort or stress that is felt when performing a variety of memory tasks; Anxiety; 12), locus of control in memory abilities (that is, the degree to which one believes that memory performance is under one's own control rather than attributable to external factors; Locus; 7), memory and achievement motivation (that is, the perceived importance of having a good memory and performing well on memory tasks; Achievement; 7), and frequency of use of external versus internal memory strategies (memory strategies are techniques aimed to support memory performance; internal memory strategies refer to the use of mental techniques, such as imagery or rehearsal, and external memory strategies refer to the use of external memory aids, such as calendars or notes; External Strategy vs. Internal Strategy; eight each).

The participants were asked to rate the statements on a 5-point Likert scale $(5=$ strongly agree; 1 = strongly disagree). We divided the total score for each scale by the number of items contained in the scale, resulting in a range of one to five for each scale (cf. McDonald-Miszczak et al., 1999).

\section{Procedure}

Each participant was tested individually in a laboratory session. First, participants gave informed consent, provided sociodemographic information, and filled in the PRMQ. Then, the instructions for the n-back task were given, followed by practice trials of the n-back task. Participants performed the n-back task twice more in concert with an event-based or a time-based PM task (the order of the two PM tasks was counterbalanced across participants). Participants were instructed for the specific PM task and were then required to repeat all their tasks in order to check for understanding. Thereafter, a distracter task was given. After this delay of approximately five minutes, the experimenter started the first n-back/PM task. After completion, retrospective memory for the embedded PM task was obtained. Thereafter, a break of 20 minutes followed during which the MIA was completed. Then, the experimenter continued with the procedure of the second PM task that was identical to the first PM task with the exception of the type of the PM task. At the end of the procedure, participants were debriefed. 


\section{RESULTS}

Ongoing Task Performance. The mean number of correct n-back task responses was $112.79(S D=5.45)$ for the event-based and $111.90(S D=6.64)$ for the time-based PM task. Ongoing task performance was positively correlated across the two types of PM tasks, $r=.79, p<.001$.

PM Performance. The mean number of PM hits was $M=4.70(S D=.72)$ for the event-based and $M=3.76(S D=1.50)$ for the time-based task. Performance in the two types of PM tasks was not correlated, $r=.13, p=.243$. The reliability of the PM performance measures was .65 for the event-based task and .74 for the time-based task (Guttman's $\lambda$ ).

PRMQ and Its Relation to PM Performance. The reliability of the PRMQ in terms of Cronbach's alpha was .86 for the Total scale, .79 for the PM Scale, and .72 for the RM Scale. Participants reported significantly higher scores on the PM Scale $(M=$ $18.16, S D=4.20)$ than on the RM Scale $(M=16.48, S D=3.84), t(86)=5.06, p<$ .001 . To examine whether the PRMQ scores may predict actual performance in the laboratory PM tasks, correlational analyses were performed. Importantly, only the PM subscale was a significant predictor for laboratory-based PM performance (time-based PM: $r=-.22, p<.050$; for event-based PM this relation approached significance: $r=-$ $.19, p=.081$ ), but not the PRMQ Total scale and the RM subscale.

MIA and Its Relation to PM Performance. The reliability of the MIA in terms of Cronbach's alpha was .74 for Task, .76 for Capacity, .90 for Change, .77 for Anxiety, .66 for Locus, .60 for Achievement, .51 for External Strategy, and .73 for Internal Strategy. To examine whether the MIA scores are associated with actual performance in the laboratory PM tasks, correlational analyses were performed. A higher number of event-based PM hits was significantly associated solely with the Capacity scale $(r=$ $.24, p<.050$ ), indicating that participants who regarded themselves as more capable in memory situations also performed better in the event-based PM task relative to individuals who rated their memory abilities as worse. With regard to time-based PM hits, only the Change scale had a significant relation to PM performance $(r=.26, p<.050)$, indicating that participants who perceived their memory abilities as more stable across the last ten years performed better in the time-based PM tasks relative to participants who reported a decline in memory ability. All the other relationships between the MIA scales and event-based or time-based PM performance were not statistically significant and were relatively low.

Cross-validation of the PRMQ. The correlation coefficients regarding the relationship between the PRMQ scales and the seven scales of the MIA are shown in Table 1.

As can be seen from Table 1, a consistent pattern across all three examined PRMQ scales emerged for the MIA scales of Capacity, Change, and Anxiety: Participants reporting high frequency of PM or RM errors in everyday life also reported having 
TABLE 1

Relationship between PRMQ subscales and MIA subscales

\begin{tabular}{llccc}
\hline Subscale of MIA & & $\begin{array}{c}\text { PRMQ } \\
\text { Total Scale }\end{array}$ & $\begin{array}{c}\text { PRMQ } \\
\text { PM Scale }\end{array}$ & $\begin{array}{c}\text { PRMQ } \\
\text { RM Scale }\end{array}$ \\
\hline Task & $r(p)$ & $-.08(.440)$ & $-.02(.885)$ & $-.15(.180)$ \\
Capacity & $r(p)$ & $-.52(<.001)$ & $-.44(<.001)$ & $-.53(<.001)$ \\
Change & $r(p)$ & $-.49(<.001)$ & $-.43(<.001)$ & $-.48(<.001)$ \\
Anxiety & $r(p)$ & $\mathbf{. 4 5}(<.001)$ &. $\mathbf{3 9}(<.001)$ & $\mathbf{. 4 4}(<.001)$ \\
Locus & $r(p)$ & $-.13(.233)$ & $-.10(.339)$ & $-.14(.207)$ \\
Achievement & $r(p)$ & $-.19(.080)$ & $-.11(.305)$ & $-.24(<.050)$ \\
External Strategy & $r(p)$ & $-.06(.579)$ & $.03(.753)$ & $-.15(.154)$ \\
Internal Strategy & $r(p)$ & $.18(.104)$ & $\mathbf{. 2 4}(<.050)$ & $.08(.473)$ \\
\hline
\end{tabular}

Notes. Significant correlations are printed in bold. All $p$-values are two-tailed.

Higher scores in the PRMQ scales indicate a higher incidence of everyday PM or RM memory errors.

Higher scores in the MIA scales indicate better knowledge of basic memory processes (Task), higher perceived memory capacities (Capacity), higher stability of memory abilities over the last ten years (Change), higher anxiety related to memory-task situations (Anxiety), higher perceived sense of control over memory (Locus), higher perceived importance of performing well in memory tasks (Achievement), and more frequent use of external or internal memory strategies, respectively (External Strategy \& Internal Strategy).

low memory abilities in the MIA. Moreover, the participants reporting high frequency of PM or RM errors subjectively perceived a decline in memory abilities over the past ten years and reported being more anxious in memory situations. Additionally, participants reporting high frequency of everyday PM errors indicated using more internal memory strategies (that is, mental techniques aimed to support memory performance, such as imagery or rehearsal), and participants reporting high frequency of RM errors in everyday life rated the importance of having a good memory and performing well in memory tasks as relatively low.

\section{DISCUSSION}

The first goal of the present study was to explore the relationship between individual scores in the recently developed PRMQ and objective measures of actual PM test performance to further test the validity of the PRMQ in predicting actual PM performance. For this purpose, we correlated the scores of the PRMQ with performance scores of an event-based and a time-based PM task carried out in the laboratory. Since performance in the two types of PM tasks was not correlated, it seemed reasonable to examine each PM task type separately instead of relating the PRMQ scores to a single measure of PM performance collapsed across the types of PM tasks.

The results revealed that the number of self-reported PM errors was marginally significantly associated with event-based PM hits, whereas the relationship between event-based PM hits and the RM Scale did not approach significance. In the timebased PM task, a higher number of self-reported PM errors was significantly associated with a higher number of time-based PM hits. By contrast, the RM score was not 
related to performance in the time-based PM task providing evidence for a differential sensitivity of the PM Scale and the RM Scale in the prediction of PM performance.

In terms of effect sizes, the size of the present correlation coefficients reflecting the relationship between PRMQ scores and PM performance are comparable to those reported by Mäntylä (2003) for a single-trial PM task. Taken together, the findings provide evidence that there indeed exists a reliable relationship between the PRMQ scores and actual PM performance. The present results differ from the findings of Mäntylä, however, by showing that the different subscales of the PRMQ may indeed have differential sensitivity in the prediction of actual PM performance, as would be indicated by the proposed structure of the PRMQ. Specifically, in contrast to the findings of Mäntylä, the present data show that the subscales of the PRMQ tapping PM may be predictors of actual PM performance, but not the subscales of the PRMQ tapping RM. In sum, the present study extends the evidence for the validity of the PRMQ and provides first data on its utility in differentiating between actual PM and RM task performance.

As a second aspect, the present study provided cross-validation data for the PRMQ. A relatively consistent pattern emerged across the PRMQ and the MIA scales; and the correlations showed a conceptually appealing pattern as mostly memory capacity, memory change, and anxiety seem to contribute to the PRMQ judgments. Thus, feelings of having good memory abilities converged with self-reports of fewer everyday PM and RM memory errors as indicated by the PRMQ scores. A perceived decline in memory ability over the last ten years was associated with a relatively high current prevalence of everyday PM and RM errors. Reports of participants of failing relatively frequently in everyday PM and RM memory situations were closely correlated with reports of being more anxious in memory-task situations.

Additionally, the MIA scale indicating subjective achievement motivation in memory tasks was significantly correlated with the RM Scale of the PRMQ, indicating that participants who rated the importance of having a good memory as relatively low also exhibit relatively poor everyday RM performance. We speculate that RM abilities are influenced by the degree to which someone is motivated to succeed in such tasks, whereas the success in everyday PM tasks is not modulated by achievement motivation but rather by other factors, such as the importance attributed to a specific intended action and its outcome (see, for example, Kliegel et al., 2001), rather than the importance that is attributed to generally being good in memory tasks.

Finally, the MIA scale assessing the use of internal memory strategies converged with the PM Scale of the PRMQ, indicating that internal memory strategies are used to a relatively high degree by participants reporting a high incidence of everyday PM errors but not by participants reporting RM problems in everyday life. Perhaps participants apply internal memory strategies when trying to prevent failures in prospective remembering, but are not aware of potential internal memory strategies that might support retrospective remembering in everyday life. Besides these distinct and close relations between the PRMQ and the MIA, there was also evidence of discriminant validity between the PRMQ subscales and the MIA scales, as general knowledge of basic memory processes, the perceived locus of control in memory abilities, and the 
use of external memory strategies were not correlated with the incidence of everyday memory problems, as assessed by the PRMQ. In conclusion, the present study firstly provides cross-validation of the PRMQ by showing close relationships with distinct scales of the MIA.

Regarding the question whether the MIA can also predict actual performance in PM tasks, the results showed that participants who regarded themselves as more capable in memory situations performed better in the event-based PM task. The results also revealed that participants who perceived their memory abilities as more stable across the last ten years performed better in the time-based PM task.

This pattern of results complements initial reports indicating that PM performance may be related to individual differences in distinct aspects of metamemory (McDonaldMiszczak et al., 1999). However, most of the MIA scales were not significantly related to PM performance. One reason for this pattern might be that the MIA more strongly refers to everyday RM rather than PM and thus cannot predict performance in PM tasks in significant ways. Indeed, as indicated by the present investigation of the PRMQ and its relation to actual task performance, self-reports tapping RM might be less closely related to performance-based PM measures than self-reports tapping PM. By consequence, the present findings highlight the utility of the PRMQ as the first self-report measure explicitly tapping both RM and PM, providing a distinction between these two types of everyday memory abilities.

In conclusion, the present study supports the idea that the PRMQ is valid in the sense that it can predict actual PM performance by its separate PM subscale, with an accuracy beyond that expected by chance, however, the RM subscale is unable to do so. Hence, based on the present and Mäntylä's (2003) findings, we suggest that the PRMQ should be considered a reliable and valid instrument for assessing everyday PM and RM performance among healthy individuals or clinical patients reporting diminished cognitive abilities. An avenue for further studies might be to extend the examination of the PRMQ's validity by administering naturalistic PM and RM tasks rather than laboratory tasks, that is, memory tasks that are carried out in everyday life. By this means, it could further be tested whether the PRMQ is a valid instrument for assessing everyday PM and RM performance (Mäntylä, 2003).

\section{NOTES}

We would like to thank Heidi Wegmüller and Tsering Bamert for their assistance in data collection and Adrian Imfeld for computer programming.

Address for correspondence: Matthias Kliegel, Department of Psychology, University of Zurich, Binzmühlestrasse 14/24, CH-8050 Zurich, Switzerland. E-mail: m.kliegel@psychologie.unizh.ch.

\section{REFERENCES}

Baddeley, A.D. (1990). Human memory: Theory and practice. Hove: Lawrence Erlbaum Associates.

Brandimonte, M.A., Einstein, G.O., \& McDaniel, M.A. (1996). Prospective memory: Theory and applications. Mahwah, NJ: Lawrence Erlbaum Associates Inc.

Crawford, J.R., Henry, J.D., Ward, A.L., \& Blake, J. (2006). The Prospective and Retrospective Memory Questionnaire (PRMQ): Latent structure, normative data and discrepancy analysis for proxy-ratings. British Journal of Clinical Psychology, 45, 83-104. 
Crawford, J.R., Smith, G., Maylor, E.A., Della Sala, S., \& Logie, R.H. (2003). The normative data and latent structure in a large non-clinical sample. Memory, 11, 261-275.

Dixon, R.A. (1989). Questionnaire research on metamemory and aging: Issues of structure and function. In L.W. Poon, D.C. Rubin, \& B.A. Wilson (Eds.), Everyday cognition in adulthood and late life (pp. 394 415). Cambridge, UK: Cambridge University Press.

Dixon, R.A. \& Hultsch, D.F (1984). The Metamemory in Adulthood (MIA) Instrument. Psychological Documents, 14,3 .

Dixon, R.A., Hultsch, D.F., \& Hertzog, C. (1988). The Metamemory in Adulthood (MIA) Questionnaire. Psychopharmacology Bulletin, 24, 671-688.

d'Ydewalle, G., Bouckaert, D., \& Brunfaut, E. (2001). Age-related differences and complexity of ongoing activities in time-and event-based prospective memory. American Journal of Psychology, 114, 411423.

Einstein, G.O., McDaniel, M.A., Richardson, S.L., Guynn, M.J., \& Cunfer, A.R. (1995). Aging and prospective memory: Examining the influences of self-initiated retrieval processes. Journal of Experimental Psychology: Learning, Memory, and Cognition, 21, 996-1007.

Ellis, J. \& Kvavilashvili, L. (2000). Prospective memory in 2000: Past, present, and future directions. Applied Cognitive Psychology, 14, S1-S9.

Hultsch, D.F., Hertzog, C., Dixon, R.A., \& Davidson, H. (1988). Memory self-knowledge and self-efficacy in the aged. In M.L. Howe \& C.J. Brainerd (Eds.), Cognitive development in adulthood: Progress in cognitive development research (pp. 65-92). New York: Springer.

Kliegel, M., Jäger, T., Phillips, L.H., Federspiel, E., Imfeld, A., Keller, M., \& Zimprich, D. (2005). Effects of sad mood on time-based prospective memory. Cognition and Emotion, 19, 1199-1213.

Kliegel, M., Martin, M., McDaniel, M.A., \& Einstein, G.O. (2001). Varying the importance of a prospective memory task: Differential effects across time-and event-based prospective memory. Memory, 9 , $1-11$.

Mäntylä, T. (2003). Assessing absentmindedness: Prospective memory complaint and impairment in middleaged adults. Memory \& Cognition, 31, 15-25.

Maylor, E.A. (1993). Aging and forgetting in prospective and retrospective memory tasks. Psychology and Aging, 8, 420-428.

Maylor, E.A. (1996). Age-related impairment in an event-based prospective-memory task. Psychology and Aging, 11, 74-78.

McDonald-Miszczak, L., Gould, O.N., \& Tychynski, D. (1999). Metamemory predictors of prospective and retrospective memory performance. The Journal of General Psychology, 126, 37-52.

Ponds, R.W.H.M. \& Jolles, J. (1999). The abridged Dutch Metamemory in Adulthood (MIA) Questionnaire: Structure and effects of age, sex, and education. Psychology and Aging, 11, 324-332.

Smith, G., Della Sala, S., Logie, R.H., \& Maylor, E.A. (2000). Prospective and retrospective memory in normal ageing and dementia: A questionnaire study. Memory, 8, 311-321.

Snodgrass, J.G. \& Vanderwart, M. (1980). A standardized set of 260 pictures: Norms for name agreement, image agreement, familiarity, and visual complexity. Journal of Experimental Psychology: Human Learning and Memory, 6, 174-215. 